\title{
Eigenvalue assignment in fractional descriptor discrete-time linear systems
}

\author{
TADEUSZ KACZOREK and KAMIL BORAWSKI
}

\begin{abstract}
The problem of eigenvalue assignment in fractional descriptor discrete-time linear systems is considered. Necessary and sufficient conditions for the existence of a solution to the problem are established. A procedure for computation of the gain matrices is given and illustrated by a numerical example.
\end{abstract} matrix.

Key words: eigenvalue assignment, fractional, descriptor, discrete-time linear system, gain

\section{Introduction}

A dynamical system is called a fractional-order system if its state equations are given by fractional-order derivative of state vector. Mathematical fundamentals of the fractional calculus are given in the $[23,25,26]$. The standard and positive fractional linear systems have been investigated in $[18,24]$ and the positive fractional linear electrical circuits in [20]. Some recent interesting results in the fractional systems theory and its applications can be found in $[8,27,28,30]$.

Descriptor (singular) linear systems were considered in many papers and books [1-7, $9-11,17,18,22,29,31]$. The positive standard and descriptor systems and their stability have been analyzed in [13-16, 28]. Descriptor positive discrete-time and continuoustime nonlinear systems have been analyzed in [10] and the positivity and linearization of nonlinear discrete-time systems by state-feedbacks in [14]. New stability tests of positive standard and fractional linear systems have been investigated in [12]. The controllability of dynamical systems has been investigated in [21].

In this paper the eigenvalue assignment problem for fractional descriptor discretetime linear systems will be investigated and procedure for computation of the statefeedback gain matrices will be presented.

The paper is organized as follows. In section 2 the problem of eigenvalue assignment in fractional descriptor discrete-time linear systems is formulated. In section 3 the

The authors is with Bialystok University of Technology, Faculty of Electrical Engineering, Wiejska 45D, 15-351 Bialystok, Poland. E-mail: kaczorek@ee.pw.edu.pl

This work was supported by National Science Centre in Poland under work No. 2014/13/B/ST7/03467.

Received 18.08.2016. 
problem is solved and procedure for computation of the state-feedback gain matrices is presented. Concluding remarks are given in section 4.

The following notation will be used: $\mathfrak{R}$ - the set of real numbers, $\Re^{n \times m}-$ the set of $n \times m$ real matrices and $\Re^{n}=\Re^{n \times 1}, I_{n}$ - the $n \times n$ identity matrix, $Z_{+}$- the set of nonnegative integers.

\section{Problem formulation}

Consider the descriptor discrete-time linear system

$$
E \Delta^{\alpha} x_{k+1}=A x_{k}+B u_{k}, \quad k \in Z_{+}=\{0,1, \ldots\}
$$

where $x_{k} \in \mathfrak{R}^{n}, u_{k} \in \mathfrak{R}^{m}$ are the state and input vectors and $E, A \in \mathfrak{R}^{n \times n}, B \in \mathfrak{R}^{n \times m}$. The fractional difference of the order $\alpha$ is defined by

$$
\Delta^{\alpha} x_{k}=\sum_{i=0}^{k}(-1)^{k}\left(\begin{array}{c}
\alpha \\
i
\end{array}\right) x_{k-i}, \quad\left(\begin{array}{c}
\alpha \\
i
\end{array}\right)=\left\{\begin{array}{cl}
1 & \text { for } \quad i=0, \\
\frac{\alpha(\alpha-1) \ldots(\alpha-i+1)}{i !} & \text { for } \quad i=1,2, \ldots
\end{array}\right.
$$

Substituting (2) into (1) yields

$$
E x_{k+1}=A_{\alpha} x_{k}+\sum_{i=1}^{k+1} c_{i} E x_{k-i+1}+B u_{k}
$$

where

$$
A_{\alpha}=A+\alpha E, \quad c_{i}=(-1)^{i}\left(\begin{array}{c}
\alpha \\
i+1
\end{array}\right), \quad i=1,2, \ldots .
$$

It is assumed that $\operatorname{rank} E=r<n$ and $\operatorname{rank} B=m$. In practical problems it is also assumed that $i$ is bounded by natural number $h=k+1>n$. We may write the equation (3) in the form

$$
\bar{E} \bar{x}_{k+1}=\bar{A} \bar{x}_{k}+\bar{B} u_{k}
$$


where

$$
\begin{aligned}
\bar{A} & =\left[\begin{array}{cccccc}
A_{\alpha} & c_{1} E & c_{2} E & \cdots & c_{h-1} E & c_{h} E \\
I_{n} & 0 & 0 & \cdots & 0 & 0 \\
0 & I_{n} & 0 & \cdots & 0 & 0 \\
\vdots & \vdots & \vdots & \ddots & \vdots & \vdots \\
0 & 0 & 0 & \cdots & I_{n} & 0
\end{array}\right] \in \Re^{\bar{n} \times \bar{n}}, \quad \bar{B}=\left[\begin{array}{c}
B \\
0 \\
0 \\
\vdots \\
0
\end{array}\right] \in \mathfrak{R}^{\bar{n} \times m}, \\
\bar{E} & =\left[\begin{array}{ccccc}
E & 0 & 0 & \cdots & 0 \\
0 & I_{n} & 0 & \cdots & 0 \\
0 & 0 & I_{n} & \cdots & 0 \\
\vdots & \vdots & \vdots & \ddots & \vdots \\
0 & 0 & 0 & \cdots & I_{n}
\end{array}\right] \in \mathfrak{R}^{\bar{n} \times \bar{n}}, \bar{x}_{k}=\left[\begin{array}{c}
x_{k} \\
x_{k-1} \\
x_{k-2} \\
\vdots \\
x_{k-h}
\end{array}\right] \in \mathfrak{R}^{\bar{n}}, \quad k \in Z_{+}, \bar{n}=n(h+1) .
\end{aligned}
$$

Let us consider the system (1) with the state-feedback

$$
\bar{u}_{k}=K_{1} \bar{x}_{k+1}+K_{2} \bar{x}_{k}
$$

where $\bar{u}_{k} \in \mathfrak{R}^{m}$ is a new input vector and $K_{1}, K_{2} \in \Re^{m \times \bar{n}}$ are gain matrices. Substitution of (7) into (5) yields

$$
\left(\bar{E}-\bar{B} K_{1}\right) \bar{x}_{k+1}=\left(\bar{A}+\bar{B} K_{2}\right) \bar{x}_{k} .
$$

The problem can be stated as follows. Given $E, A, B, \alpha \in(0,1)$ find $K_{1}, K_{2}$ such that the closed-loop system has desired eigenvalues $z_{1}, z_{2}, \ldots, z_{n},\left|z_{k}\right|<1, k=1, \ldots n$.

\section{Problem solution}

The problem will be solved by the use of the following two steps procedure.

Step 1. (Subproblem 1) Find $K_{1}$ such that $\bar{E}-\bar{B} K_{1}=I_{\bar{n}}$.

Step 2. (Subproblem 2) Find $K_{2}$ such that $\bar{A}+\bar{B} K_{2}$ has desired eigenvalues.

The first subproblem has a solution if and only if [3]

$$
\operatorname{rank}\left[\begin{array}{cc}
\bar{E} & \bar{B}
\end{array}\right]=\bar{n}, \quad \operatorname{rank} \bar{B}=m
$$

Theorem 8 If the conditions (9) are satisfied then the equation

$$
\bar{E}-\bar{B} K_{1}=I_{\bar{n}}
$$


has the solution

$$
K_{1}=\left\{\left[\bar{B}^{T} \bar{B}\right]^{-1} \bar{B}^{T}+K\left[I_{\bar{n}}-\bar{B}\left[\bar{B}^{T} \bar{B}\right]^{-1} \bar{B}^{T}\right]\right\}\left(\bar{E}-I_{\bar{n}}\right),
$$

where $K$ is an arbitrary matrix.

Proof From (10) we have

$$
\bar{B} K_{1}=\bar{E}-I_{\bar{n}} .
$$

If conditions (9) are met then there exists the left pseudoinverse of the matrix $\bar{B}$ given by the formula [19]

$$
\bar{B}_{L}=\left[\bar{B}^{T} \bar{B}\right]^{-1} \bar{B}^{T}+K\left[I_{\bar{n}}-\bar{B}\left[\bar{B}^{T} \bar{B}\right]^{-1} \bar{B}^{T}\right]
$$

and

$$
K_{1}=\bar{B}_{L}\left(\bar{E}-I_{\bar{n}}\right)=\left\{\left[\bar{B}^{T} \bar{B}\right]^{-1} \bar{B}^{T}+K\left[I_{\bar{n}}-\bar{B}\left[\bar{B}^{T} \bar{B}\right]^{-1} \bar{B}^{T}\right]\right\}\left(\bar{E}-I_{\bar{n}}\right),
$$

which is equivalent to (11).

Remark 1 In particular case when $K=0$ we have

$$
K_{1}=\left[\bar{B}^{T} \bar{B}\right]^{-1} \bar{B}^{T}\left(\bar{E}-I_{\bar{n}}\right)=\left[\left[B^{T} B\right]^{-1} B^{T}\left(E-I_{n}\right) \quad 0 \quad \cdots \quad 0\right]
$$

and then

$$
K_{1} \bar{x}_{k+1}=\left[B^{T} B\right]^{-1} B^{T}\left(E-I_{n}\right) x_{k+1} .
$$

The second subproblem will be solved substituting (10) into (8). Thus we have

$$
\bar{x}_{k+1}=\left(\bar{A}+\bar{B} K_{2}\right) \bar{x}_{k} .
$$

Theorem 9 There exists a matrix $K_{2}$ such that the matrix $\bar{A}+\bar{B} K_{2}$ has the desired eigenvalues $\lambda_{k}, k=1, \ldots, \bar{n}$ if and only if the pair $(\bar{A}, \bar{B})$ is controllable.

Proof The proof is given in [11].

To solve the problem one of the well-known methods [11] can be applied. To simplify the notation we consider the single-input system (17) with a controllable pair $(\bar{A}, \bar{B})$. Following [11] there exists a matrix

$$
P=\left[\begin{array}{c}
p_{1} \\
p_{1} \bar{A} \\
\vdots \\
p_{1} \bar{A}^{\bar{n}-1}
\end{array}\right]
$$


that transforms every controllable pair $(\bar{A}, \bar{B})$ to the canonical form

$$
\widetilde{A}=P \bar{A} P^{-1}=\left[\begin{array}{ccccc}
0 & 1 & 0 & \cdots & 0 \\
0 & 0 & 1 & \cdots & 0 \\
\vdots & \vdots & \vdots & \ddots & \vdots \\
0 & 0 & 0 & \cdots & 1 \\
-\widetilde{a}_{0} & -\widetilde{a}_{1} & -\widetilde{a}_{2} & \cdots & -\widetilde{a}_{\bar{n}-1}
\end{array}\right], \widetilde{B}=P \bar{B}=\left[\begin{array}{c}
0 \\
0 \\
\vdots \\
0 \\
1
\end{array}\right] .
$$

The vector $p_{1}$ in (18) is the $\bar{n}$-th row of the matrix

$$
\left[\begin{array}{llll}
\bar{B} & \bar{A} \bar{B} & \cdots & \bar{A}^{\bar{n}-1} \bar{B}
\end{array}\right]^{-1} .
$$

The characteristic polynomial of the matrix $\widetilde{A}$ has the form

$$
\operatorname{det}\left[I_{\bar{n}} z-\widetilde{A}\right]=z^{\bar{n}}+\widetilde{a}_{\bar{n}-1} z^{\bar{n}-1}+\ldots+\widetilde{a}_{1} z+\widetilde{a}_{0}
$$

and the characteristic polynomial of the closed-loop system matrix $\widetilde{A}+\widetilde{B} K_{2}$ has the form

$$
\operatorname{det}\left[I_{\bar{n}} z-\widetilde{A}-\widetilde{B} K_{2}\right]=z^{\bar{n}}+\widetilde{d}_{\bar{n}-1} z^{\bar{n}-1}+\ldots+\widetilde{d}_{1} z+\widetilde{d}_{0} .
$$

The matrix satisfying (22) is given by

$$
K_{2}=\left[\begin{array}{lllll}
\widetilde{d}_{0}-\widetilde{a}_{0} & \widetilde{d}_{1}-\widetilde{a}_{1} & \cdots & \widetilde{d}_{\bar{n}-1}-\widetilde{a}_{\bar{n}-1}
\end{array}\right] .
$$

The considerations can be easily extended to multi-input systems [11].

From the above we have the following procedure.

\section{Procedure 1.}

Step 1. Knowing $A, B, E, \alpha$ choose $h>n$ and compute the matrices $\bar{A}, \bar{B}, \bar{E}$ defined by (6).

Step 2. Check the conditions (9), then using $\bar{E}$ and $\bar{B}$ compute $K_{1}$ defined by (11). In particular case when $K=0$ we can use matrices $E$ and $B$ (see (15)).

Step 3. Applying one of the well-known methods [11] and using $\bar{A}, \bar{B}$ compute $K_{2}$ such that the matrix $\bar{A}+\bar{B} K_{2}$ has the desired eigenvalues $\lambda_{k}, k=1, \ldots, \bar{n}$, $\operatorname{Re} \lambda_{k}<0$. The method for single-input systems presented above can be used.

Example 1 Consider the fractional descriptor discrete-time linear system (1) with the matrices

$$
E=\left[\begin{array}{lll}
1 & 0 & 0 \\
0 & 1 & 0 \\
0 & 0 & 0
\end{array}\right], A=\left[\begin{array}{lll}
0 & 1 & 0 \\
0 & 0 & 1 \\
1 & 0 & 0
\end{array}\right], B=\left[\begin{array}{l}
0 \\
0 \\
1
\end{array}\right]
$$


and $\alpha=0.5$. Find $K_{1}$ and $K_{2}$ such that the closed-loop system has the eigenvalues $\lambda_{k}=0, k=1, \ldots, 9$. Using the Procedure 1 we obtain the following.

Step 1. Step 1. We choose $h=2$. From (6) we have

$$
\begin{gathered}
\bar{A}=\left[\begin{array}{ccccccccc}
0.5 & 1 & 0 & 0.125 & 0 & 0 & 0.0625 & 0 & 0 \\
0 & 0.5 & 1 & 0 & 0.125 & 0 & 0 & 0.0625 & 0 \\
1 & 0 & 0.5 & 0 & 0 & 0 & 0 & 0 & 0 \\
1 & 0 & 0 & 0 & 0 & 0 & 0 & 0 & 0 \\
0 & 1 & 0 & 0 & 0 & 0 & 0 & 0 & 0 \\
0 & 0 & 1 & 0 & 0 & 0 & 0 & 0 & 0 \\
0 & 0 & 0 & 1 & 0 & 0 & 0 & 0 & 0 \\
0 & 0 & 0 & 0 & 1 & 0 & 0 & 0 & 0 \\
0 & 0 & 0 & 0 & 0 & 1 & 0 & 0
\end{array}\right] \\
\bar{E}=\left[\begin{array}{lllllllll}
1 & 0 & 0 & 0 & 0 & 0 & 0 & 0 & 0 \\
0 & 1 & 0 & 0 & 0 & 0 & 0 & 0 & 0 \\
0 & 0 & 0 & 0 & 0 & 0 & 0 & 0 & 0 \\
0 & 0 & 0 & 1 & 0 & 0 & 0 & 0 & 0 \\
0 & 0 & 0 & 0 & 1 & 0 & 0 & 0 & 0 \\
0 & 0 & 0 & 0 & 0 & 1 & 0 & 0 & 0 \\
0 & 0 & 0 & 0 & 0 & 0 & 1 & 0 & 0 \\
0 & 0 & 0 & 0 & 0 & 0 & 0 & 1 & 0 \\
0 & 0 & 0 & 0 & 0 & 0 & 0 & 0 & 1
\end{array}\right], \bar{B}=\left[\begin{array}{l}
0 \\
0 \\
1 \\
0 \\
0 \\
0 \\
0 \\
0 \\
0
\end{array}\right]
\end{gathered}
$$

Step 2. The conditions (9) are satisfied. Using (25) with (11) for $K=\left[\begin{array}{lllllllll}1 & 0 & 0 & 0 & 0 & 0 & 0 & 0 & 0\end{array}\right]$ we obtain the first gain matrix

$$
K_{1}=\left[\begin{array}{lllllllll}
0 & 0 & -1 & 0 & 0 & 0 & 0 & 0 & 0
\end{array}\right] .
$$

It is easy to check that $\bar{E}-\bar{B} K_{1}=I_{9}$.

Step 3. Step 3. Using the presented algorithm for single-input systems we compute the matrix 


$$
\left[\begin{array}{llll}
\bar{B} & \bar{A} \bar{B} & \cdots & \bar{A}^{\bar{n}-1} \bar{B}
\end{array}\right]^{-1}=
$$

$$
=\left[\begin{array}{ccccccccc}
0 & 0 & 1 & -1 & 0 & -0.5 & 0 & 0 & 0 \\
0 & 0 & 0 & 0 & 0 & -1 & -1 & 0 & -0.5 \\
-13.5 & 0.5 & 0 & 5.5 & 10.5 & -0.5 & 2.4 & 3.1 & 5.4 \\
54 & 52 & 0 & -48 & -96 & -52 & 3.3 & 22.7 & 9.2 \\
82 & 370 & 0 & -2 & -248 & -370 & -49.3 & -104.8 & -29.2 \\
-688 & -1656 & 0 & 376 & 1776 & 1656 & 114 & 84 & -174 \\
384 & 1056 & 0 & -160 & -984 & -1056 & -104 & -156 & 24 \\
-1024 & -2368 & 0 & 576 & 2560 & 2368 & 160 & 112 & 224 \\
640 & 1408 & 0 & -384 & -1600 & -1408 & -80 & -16 & 176
\end{array}\right]
$$

The vector has the form

$$
p_{1}=\left[\begin{array}{lllllllll}
640 & 1408 & 0 & -384 & -1600 & -1408 & -80 & -16 & 176
\end{array}\right] .
$$

Using (18) we compute the matrix

$$
P=\left[\begin{array}{ccccccccc}
640 & 1408 & 0 & -384 & -1600 & -1408 & -80 & -16 & 176 \\
-64 & -256 & 0 & 0 & 160 & 256 & 40 & -88 & 40 \\
-32 & -32 & 0 & 32 & 56 & 32 & -4 & -16 & -4 \\
16 & 8 & 0 & -8 & -20 & -8 & -2 & -2 & -2 \\
0 & 0 & 0 & 0 & -1 & 0 & 1 & 0.5 & 1 \\
0 & -1 & 0 & 1 & 0.5 & 1 & 0 & 0 & 0 \\
1 & 0 & 0 & 0 & -0.1 & 0 & 0 & -0.1 & 0 \\
0.5 & 0.9 & 0 & 0.1 & -0.1 & 0.1 & 0.1 & 0 & 0.1 \\
0.4 & 0.9 & 1 & 0.1 & 0.1 & 0.1 & 0 & 0.1 & 0
\end{array}\right]
$$

which transforms the pair $(\bar{A}, \bar{B})$ to the canonical form (see (19))

$$
\widetilde{A}=\left[\begin{array}{ccccccccc}
0 & 1 & 0 & 0 & 0 & 0 & 0 & 0 & 0 \\
0 & 0 & 1 & 0 & 0 & 0 & 0 & 0 & 0 \\
0 & 0 & 0 & 1 & 0 & 0 & 0 & 0 & 0 \\
0 & 0 & 0 & 0 & 1 & 0 & 0 & 0 & 0 \\
0 & 0 & 0 & 0 & 0 & 1 & 0 & 0 & 0 \\
0 & 0 & 0 & 0 & 0 & 0 & 1 & 0 & 0 \\
0 & 0 & 0 & 0 & 0 & 0 & 0 & 1 & 0 \\
0 & 0 & 0 & 0 & 0 & 0 & 0 & 0 & 1 \\
0 & 0 & -0.002 & -0.0117 & -0.0234 & -0.0781 & 1.125 & -0.5 & 1.5
\end{array}\right],
$$




$$
\widetilde{B}=\left[\begin{array}{lllllllll}
0 & 0 & 0 & 0 & 0 & 0 & 0 & 0 & 1
\end{array}\right]^{T} .
$$

Using (23) we have the second gain matrix

$$
K_{2}=\left[\begin{array}{lllllllll}
0 & 0 & -0.002 & -0.0117 & -0.0234 & -0.0781 & 1.125 & -0.5 & 1.5
\end{array}\right] .
$$

The closed-loop system matrix is given by

$$
\widetilde{A}+\widetilde{B} K_{2}=\left[\begin{array}{ccccccccc}
0 & 1 & 0 & 0 & 0 & 0 & 0 & 0 & 0 \\
0 & 0 & 1 & 0 & 0 & 0 & 0 & 0 & 0 \\
0 & 0 & 0 & 1 & 0 & 0 & 0 & 0 & 0 \\
0 & 0 & 0 & 0 & 1 & 0 & 0 & 0 & 0 \\
0 & 0 & 0 & 0 & 0 & 1 & 0 & 0 & 0 \\
0 & 0 & 0 & 0 & 0 & 0 & 1 & 0 & 0 \\
0 & 0 & 0 & 0 & 0 & 0 & 0 & 1 & 0 \\
0 & 0 & 0 & 0 & 0 & 0 & 0 & 0 & 1 \\
0 & 0 & 0 & 0 & 0 & 0 & 0 & 0 & 0
\end{array}\right]
$$

and has desired eigenvalues $\lambda_{k}=0, k=1, \ldots, 9$.

\section{Concluding remarks}

The problem of eigenvalue assignment in fractional descriptor discrete-time linear systems has been considered. Necessary and sufficient conditions for the existence of a solution to the problem have been established. A procedure for computation of the gain matrices has been given and illustrated by a numerical example.

The considerations can be extended to fractional descriptor continuous-time linear systems.

\section{References}

[1] R. BRU, C. COLL and E. SANChEZ: About positively discrete-time singular systems. In Mastorakis M.E. (Ed.), System and Control: Theory and Applications, World Scientific and Engineering Society, Athens, 2000, 44-48.

[2] R. Bru, C. Coll, S. Romero-Vivo and E. Sanchez: Some problems about structural properties of positive descriptor systems. In Benvenuti A., de Santis A. and Farina L. (Eds.), Positive Systems, Lecture Notes in Control and Information Sciences, 294 (2003), Springer, Berlin, 233-240. 
[3] A. Bunse-Gerstner, N. Nichols and V. Mehrmann: Regularization of descriptor systems by derivative and proportional state feedback. SIAM J. on Matrix Analysis and Applications, 13(1), (1992), 46-67.

[4] S.L. Campbell, C.D. Meyer and N.J. Rose: Applications of the Drazin inverse to linear systems of differential equations with singular constant coefficients. SIAM J. on Applied Mathematics, 31(3), (1976), 411-425.

[5] L. DAI: Singular Control Systems. Lecture Notes in Control and Information Sciences, Springer-Verlag, Berlin, 1989.

[6] M. Dodig and M. Stosic: Singular systems state feedbacks problems. Linear Algebra and its Applications, 431(8), (2009), 1267-1292.

[7] G.R. Duan: Analysis and Design of Descriptor Linear Systems. Springer, New York, 2010.

[8] A. DZIELIŃSKI, D. SiERoCIUK and G. SARWAS: Ultracapacitor parameters identification based on fractional order model. Proc. 10th European Control Conference ECC'09, Budapest, (2009).

[9] M.M. FAHMY and J. O'ReILl: Matrix pencil of closed-loop descriptor systems: Infinite-eigenvalues assignment. Int. J. of Control, 49(4), (1989), 1421-1431.

[10] T. KaCzoreK: Descriptor positive discrete-time and continuous-time nonlinear systems. Proc. of SPIE, 9290(2014), 1-11.

[11] T. KaCzorek: Linear Control Systems vol. 1, Research Studies Press, J. Wiley, New York, 1992.

[12] T. KACZOREK: New stability tests of positive standard and fractional linear systems. Circuits and Systems, 2(4), (2011), 261-268.

[13] T. KaCzoreK: Positive 1D and 2D Systems, Springer-Verlag, London, 2001.

[14] T. KaczoreK: Positivity and linearization of a class of nonlinear discrete-time systems by state feedbacks. Logistyka, 6 (2014), 5078-5083.

[15] T. KaCzoreK: Positive descriptor discrete-time linear systems. Problems of Nonlinear Analysis in Engineering Systems, 1(7), (1998), 38-54.

[16] T. KaCZOREK: Positivity and stability of discrete-time nonlinear systems. Proc. of 2nd IEEE Intern. Conf. on Cybernetics CYBCONF, (2015), Gdynia, Poland.

[17] T. Kaczorek: Positive singular discrete time linear systems. Bull. Pol. Acad. Techn. Sci., 45(4), (1997), 619-631. 
[18] T. KaCzOREK: Selected Problems of Fractional Systems Theory, Springer-Verlag, Berlin 2012.

[19] T. KaczoreK: Vectors and Matrices in Automation and Electrotechnics, WNT, Warszawa, 1998 (in Polish).

[20] T. KaCZoreK and K. Rogowski: Fractional Linear Systems and Electrical Circuits. Studies in Systems, Decision and Control, 13 Springer, 2015.

[21] J. Klamka: Controllability of dynamical systems. A survey. Bull. Pol. Acad. Techn. Sci., 61(2), (2013), 221-229.

[22] KUCERA and P. ZaGAlaK: Fundamental theorem of state feedback for singular systems. Automatica, 24(5), (1988), 653-658.

[23] K.B. Oldham and J. Spanier: The Fractional Calculus. Academic Press, New York 1974.

[24] P. OstalczyK: Discrete Fractional Calculus. World Scientific Publ. Co., New Jersey 2016.

[25] P. Ostalczyk: Epitome of the fractional calculus: Theory and its Applications in Automatics. Wydawnictwo Politechniki Łódzkiej, Łódź, 2008 (in Polish).

[26] I. Podlubny: Fractional Differential Equations. Academic Press, San Diego 1999.

[27] A.G. Radwan, A.M. Soliman, A.S. Elwakil and A. Sedeek: On the stability of linear systems with fractional-order elements. Chaos, Solitons and Fractals, 40(5), (2009), 2317-2328.

[28] E.J. Solteiro Pires, J.A. Tenreiro Machado and P.B. Moura Oliveira: Fractional dynamics in genetic algorithms. Workshop on Fractional Differentiation and its Application, 2 (2006), 414-419.

[29] P. VAN DoOREn: The computation of Kronecker's canonical form of a singular pencil. Linear Algebra and its Applications, 27 (1979), 103-140.

[30] B.M. Vinagre, C.A. Monje and A.J. CAlderon: Fractional order systems and fractional order control actions. Lecture 3 IEEE CDC'02 TW\#2: Fractional calculus Applications in Automatic Control and Robotics, (2002).

[31] E. VIRNIK: Stability analysis of positive descriptor systems. Linear Algebra and its Applications, 429(10), (2008), 2640-2659. 